\title{
Manej o da Planta Daninha Brachiaria plantaginea Resistente aos HERBICIDAS INIBIDORES DA ACCASE ${ }^{1}$
}

\author{
Management of the Weed Brachiaria plantaginea Resistant to ACCase Inhibitor Herbicides
}

\author{
CHRISTOFFOLETI, P.J. ${ }^{2}$ KEHDI, C.A. ${ }^{3}$ e CORTEZ, M.G. ${ }^{4}$
}

\begin{abstract}
RESUMO - O objetivo deste trabalho foi estudar o manejo de uma população da planta daninha capim-marmelada (Brachiaria plantaginea) resistente aos herbicidas inibidores da ACCase (ariloxifenoxipropionatos e ciclohexanodionas), utilizados na cultura da soja no sistema de plantio direto. Para isso, foram realizados dois experimentos: um utilizando herbicidas de manejo, com mecanismos de ação alternativos, comparando a eficácia destes com os inibidores da ACCase; e outro apenas com herbicidas inibidores da ACCase e aditivos misturados à calda. As sementes resistentes foram produzidas a partir de plantas comprovadamente resistentes; para comparação de resultados, foi utilizada uma população de capim-marmelada que nunca tinha sido pulverizada com inibidores da ACCase, portanto uma população suscetível. O experimento com os herbicidas de manejo foi realizado em condições de campo, porém as plantas ficaram em caixas com capacidade de $50 \mathrm{~L}$, evitando-se a disseminação destas para áreas adjacentes. Concluiu-se que as plantas resistentes não apresentam resistência múltipla com herbicidas de manejo com mecanismos de ação alternativos, podendo os herbicidas glyphosate, paraquat, sulfosate, paraquat + diuron, MSMA e glufosinate ser utilizados no período entressafra das áreas com sistema de plantio direto, para manejo de populações resistentes de capim-marmelada aos herbicidas inibidores da ACCase. O experimento com aditivos foi realizado em casa de vegetação, com plantas em vasos individuais, utilizando sementes das populações suscetiveis e resistentes. Os herbicidas utilizados foram inibidores da ACCase, em cuja calda foram adicionados aditivos nitrogenados, do tipo uréia e sulfato de amônio, além de um tratamento sem aditivos. Concluiu-se que os aditivos não alteraram a eficácia dos herbicidas inibidores da ACCase nas plantas provenientes tanto da população suscetivel quanto da resistente.
\end{abstract}

Palavras-chave: aditivos nitrogenados, herbicidas de manejo, uréia, sulfato de amônio, capim-marmelada.

\begin{abstract}
The objective of this research was to study a population of alexandergrass (Brachiaria plantaginea) resistant to ACCase inhibitor herbicides (ariloxyphenoxypropionic and cyclohexanodiones), usually sprayed on soybean under the conservation tillage system. Two experiments were conducted, one under field conditions, comparing the efficacy of nonselective herbicides to ACCase inhibitors, and another under greenhouse conditions, using ACCase inhibitors with nitrogenous additives in the spray solution. Resistant seeds were collected from a site of suspected resistant population, and compared to a population of alexandergrass that had never been sprayed with ACCase inhibitors, the susceptible population. The experiment with non-selective herbicides was conducted under field conditions, but plants were confined to pots of 50 L capacity, avoiding the dissemination of the seeds to adjacent areas. It was then concluded that resistant plants did not show multiple resistance to non selective herbicides with alternative mechanisms of action, with glyphosate, paraquat, paraquat + diuron, MSMA and
\end{abstract}

1 Recebido para publicação em 20/12/1999 e na forma revisada em 8/2/2001.

2 Eng.-Agrônomo, Prof. Associado do Departamento de Produção Vegetal da Escola Superior de Agricultura "Luiz de Queiroz", Av. Pádua Dias, 11, Caixa Postal, 9, 13418-900 Piracicaba-SP, <pjchrist@esalq.usp.br>; ${ }^{3}$ Graduando em Engenharia Agronômica, Escola Superior de Agricultura "Luiz de Queiroz"; ${ }^{4}$ Professora Adjunta da Universidade Estadual de Ponta Grossa, Dep. de Fitotecnia e Fitossanidade, Av. Carlos Cavalcanti, 4748 - Bloco "F”, 84030-000 Ponta Grossa-PR.

Planta Daninha, Viçosa-MG, v.19, n.1, p.61-66, 2001 
glufosinate alternative herbicides being possible to be used to control the weed during the winter to manage populations of alexandergrass resistant to ACCase inhibitor herbicides. The additive experiment was conducted under greenhouse conditions in pots, using seeds from the same populations used in the non selective experiment. The herbicides tested were ACCase inhibitors, and the additive treatments were ammonium sulfate and urea. It was then concluded that the additives did not enhance ACCase inhibitor herbicide efficacy in neither of the alexandergrass populations.

Key words: nitrogenous additives, non-selective herbicides, urea, ammonium sulfate, alexandergrass.

\section{INTRODUÇÃO}

O controle das plantas daninhas em determinada área ao longo dos anos por meio de um mesmo herbicida (ou de um mesmo mecanismo de ação) exerce pressão de seleção sobre a população de plantas daninhas, favorecendo o desenvolvimento de biótipos resistentes a estes herbicidas. O biótipo resistente passa a enfrentar menor competição com os suscetiveis, permitindo maior sobrevivência e aumento no número daqueles indivíduos, tornando a população resistente. Para que esse fenômeno seja evitado, é recomendada, entre outras, a rotação de herbicidas, evitando o uso do mesmo herbicida (ou de outros herbicidas com o mesmo mecanismo de ação) ao longo dos anos.

No Brasil há casos comprovados de populações de plantas daninhas resistentes em áreas que foram submetidas à mesma cultura e ao mesmo herbicida durante anos seguidos. O primeiro caso foi relatado por Christoffoleti et al. (1994) com a planta daninha picão-preto (Bidens pilosa), em regiões produtoras de soja.

Populações de Bidens pilosa e Euphorbia heterophylla resistentes aos herbicidas inibidores da ALS e de Brachiaria plantaginea resistentes aos inibidores da ACCase foram encontradas em algumas regiões produtoras de soja no Brasil (Vidal e Fleck, 1997). Ponchio (1997) também confirmou a existência de populações resistentes de picão-preto a estes produtos nos Estados do Mato Grosso do Sul e Rio Grande do Sul. Gazziero et al. (1997) e Christoffoleti et al. (1998) confirmaram a resistência de biótipos da planta daninha Brachiaria plantaginea aos herbicidas inibidores da ACCase, provenientes de uma área de cultura de soja da região sudoeste do Estado do Paraná.

Planta Daninha, Viçosa-MG, v.19, n.1, p.61-66, 2001
Embora os relatos existentes na literatura comprovem o aparecimento de biótipos resistentes de plantas daninhas no Brasil aos herbicidas inibidores da ACCase, como no caso da planta daninha capim-marmelada (Brachiaria plantaginea), até o momento poucas são as pesquisas publicadas que evidenciam formas alternativas de manejo das populações resistentes. Assim, esta pesquisa foi desenvolvida com o objetivo de estudar herbicidas não-seletivos para o manejo de populações resistentes, comparadas com suscetiveis, e o efeito de adjuvantes adicionados à calda de herbicidas inibidores da ACCase, a fim de verificar comparativamente a eficácia dos herbicidas inibidores da ACCase aplicados em biótipos resistentes e suscetiveis.

\section{MATERIAL E MÉTODOS}

\section{Herbicidas não-seletivos}

O experimento foi conduzido em caixas de cimento-amianto com capacidade de $50 \mathrm{~L}$, usando como substrato solo proveniente da camada arável de um Podzólico VermelhoEscuro, cuja análise química revelou $\mathrm{pH}$ de 5,0, matéria orgânica de $16 \mathrm{~g} \mathrm{dm}^{-3}$ e saturação de bases de $64 \%$.

As sementes da população resistente de capim-marmelada foram coletadas de uma área de produção de soja proveniente do Estado do Paraná, que comprovadamente era resistente aos herbicidas inibidores da ACCase, conforme experimentação previamente executada pelo Departamento de Produção Vegetal da ESALQ/ USP, assim como as sementes de uma população suscetivel foram coletadas em área cujo histórico de uso de herbicida não constava de nenhuma aplicação de inibidores da ACCase. 
As sementes dos dois biótipos foram inicialmente plantadas em caixas plásticas, sob condições de casa de vegetação, e as plantas obtidas foram então transplantadas para as caixas de cimento-amianto com o substrato. Foram transplantadas em cada caixa cinco plantas resistentes e cinco plantas suscetiveis aos inibidores da ACCase, dispostas em linha. As caixas foram colocadas em ambiente desprotegido e irrigadas de acordo com a necessidade para um bom desenvolvimento das plantas até o estádio de prefloração, quando foram então pulverizadas com pulverizador costal pressurizado com $\mathrm{CO}_{2}$, munido de dois bicos, com ponta XR 110 03, aplicando-se um volume de calda correspondente a $300 \mathrm{~L} \mathrm{ha}^{-1}$, com pressão de trabalho de $240 \mathrm{kPa}$. As condições ambientais foram: temperatura de $28^{\circ} \mathrm{C}$ e umidade relativa do ar no início da aplicação (9 horas) de 66\% e, no final, ( 11 horas) de $80 \%$. Os tratamentos herbicidas utilizados, com suas respectivas doses, encontram-se na Tabela 1.

Tabela 1 - Tratamentos utilizados no experimento com herbicidas não-seletivos

\begin{tabular}{|l|c|c|}
\hline \multicolumn{1}{|c|}{ Herbicidas } & $\begin{array}{c}\text { Doses } \\
\left(\mathrm{g} \text { i.a. ha }{ }^{-1}\right)\end{array}$ & $\begin{array}{c}\text { Doses } \\
\left(\text { L p.c. ha }^{-1}\right)\end{array}$ \\
\hline 1. glyphosate & 1.440 & 3,0 \\
2. paraquat & 600 & 3,0 \\
3. amônio-glufosinate & 600 & 3,0 \\
4. sulfosate & 1.440 & 3,0 \\
5. paraquat + diuron & $300+600$ & 3,0 \\
6. MSMA + diuron & $980+2.520$ & 7,0 \\
7. sethoxydim & 230 & 1,25 \\
8. fluazifop-butil & 250 & 2,0 \\
9. testemunha (sem herbicida) & & --- \\
\hline
\end{tabular}

i.a. $=$ ingrediente ativo; p.c. $=$ produto comercial.

Para quantificar o controle do capim-marmelada por meio dos herbicidas, utilizou-se escala visual, atribuindo a nota zero para nenhum controle e cem para controle total, comparados à testemunha, avaliação esta feita aos 21 dias após a aplicação (DAA). Os dados obtidos foram submetidos à análise de variância, com as médias sendo comparadas entre si pelo teste de Tukey a $5 \%$ de probabilidade.

\section{Adjuvantes}

Este experimento foi conduzido em casa de vegetação, em vasos com $500 \mathrm{~mL}$ de capacidade, utilizando como substrato um composto de duas partes de solo, uma parte de areia e duas partes de esterco curtido. Os resultados da análise química desse substrato revelou $\mathrm{pH}$ de 6,4, matéria orgânica de 47 g. $\mathrm{dm}^{-3}$ e saturação de bases de $91 \%$. As plantas transplantadas para os vasos tiveram a mesma procedência do experimento dos herbicidas de manejo.

Os tratamentos herbicidas utilizados no experimento encontram-se listados na Tabela 2 , e os herbicidas foram aplicados sobre a parte aérea das plantas com um pulverizador de laboratório acionado por um motor elétrico, ponta de pulverização do tipo leque, modelo TeeJet $8003 \mathrm{E}$, trabalhando a $50 \mathrm{~cm}$ da superfície do alvo. Os produtos químicos foram aplicados em mistura com água, em um volume de calda correspondente a $300 \mathrm{~L} \mathrm{ha}^{-1}$ e uma pressão de $275 \mathrm{kPa}$ através de ar comprimido.

Tabela 2 - Tratamentos utilizados no experimento com adjuvantes

\begin{tabular}{|l|c|c|l|}
\hline Herbicidas & $\begin{array}{c}\text { Doses } \\
\left(\text { L p.c. ha }{ }^{-1}\right)\end{array}$ & $\begin{array}{c}\text { Doses } \\
\left(\mathrm{g} \text { i.a. } \mathrm{L}^{-1}\right)\end{array}$ & \multicolumn{1}{|c|}{ Aditivos } \\
\hline sethoxydim & 1,25 & 184 & $\begin{array}{l}\text { S.A.* } \\
\text { Uréia } \\
\text { Sem aditivo }\end{array}$ \\
\hline fluazifop-butil & 2,0 & 125 & $\begin{array}{l}\text { S.A. } \\
\text { Uréia } \\
\text { Sem aditivo }\end{array}$ \\
\hline fenoxaprop-ethyl & 0,85 & 110 & $\begin{array}{l}\text { S.A. } \\
\text { Uréia } \\
\text { Sem aditivo }\end{array}$ \\
\hline testemunha & ----------- \\
\hline
\end{tabular}
* S.A. = sulfato de amônio. O S.A. e a uréia foram adicionados à
calda de pulverização na dose de 0,5\%.

As avaliações de controle foram feitas utilizando a mesma escala do experimento de herbicidas de manejo aos 7 e 14 dias após a aplicação (DAA). Os resultados foram submetidos à análise de variância, com as médias sendo comparadas entre si pelo teste de Tukey a $5 \%$ de probabilidade.

\section{RESULTADOS E DISCUSSÃO}

\section{Herbicidas não-seletivos}

Os resultados médios de controle de capimmarmelada, na avaliação realizada aos 21 DAA, indicam que todos os herbicidas controlaram 
o biótipo suscetível com eficiência acima de $80 \%$, exceto a mistura de MSMA + diuron (Tabela 3). É importante ressaltar nesses resultados que os herbicidas inibidores da ACCase sethoxydim e fluazifop também controlaram o capim-marmelada satisfatoriamente, indicando que esse biótipo utilizado no experimento era suscetivel a esse mecanismo de ação.

Dentro do biótipo resistente verifica-se, pela Tabela 3, que o glyphosate, paraquat, glufosinate, sulfosate e paraquat mais diuron controlam de forma eficiente o capim-marmelada, acima de $80 \%$, não diferindo estatisticamente do controle do biótipo suscetivel. Esse fato demonstra que o biótipo estudado apresenta resistência cruzada aos herbicidas inibidores da ACCase ariloxifenoxipropionicos (fluazifopbutil) e cicloexanodionas (sethoxydim) e não tem nenhum mecanismo de resistência múltipla aos herbicidas inibidores da fosfoenolpiruvyl shikimato sintetase (glyphosate e sulfosate), aos inibidores do fotossistema I (paraquat) e aos inibidores da fosforilação oxidativa (MSMA).

O manejo da planta daninha capim-marmelada por meio de herbicidas não-seletivos e com mecanismos de ação alternativos aos inibidores da ACCase é uma possibilidade de controle de populações resistentes desta planta daninha. De acordo com Moss (1997), o uso de herbicidas com mecanismo de ação alternativo, ou seja, mecanismo de ação diferenciado daquele cuja população foi selecionada para resistência, é uma estratégia de sucesso a ser adotada pelos produtores de soja, pelo menos a curto prazo.
O manejo de plantas daninhas, na atualidade, está fundamentado principalmente no uso de herbicidas; no entanto, métodos alternativos de controle destas plantas estão sendo deixados em segundo plano pela maioria dos agricultores (Owen, 1997). Assim, o fenômeno do aparecimento de populações resistentes de plantas daninhas a herbicidas deixa evidente a necessidade de que o manejo de plantas daninhas na agricultura seja praticado de forma integrada (Matthews, 1994).

Nas áreas onde uma agricultura conservacionista é utilizada, a seleção de populações resistentes a herbicidas seletivos aplicados em condições de pós-emergência já tem sido observada nesse sistema de cultivo (Costa, 1997). Uma das formas de manejo e prevenção de populações resistentes, neste sistema, é a alternância com herbicidas de diferentes mecanismos de ação durante o manejo das plantas daninhas através de herbicidas não-seletivos (Gill, 1997).

Uma das principais recomendações de manejo de populações resistentes em áreas agrícolas é a integração ao sistema de manejo de herbicidas com mecanismo de ação diferenciado daquele em que a população foi pressionada para resistência (Powles \& Holtum, 1994). O uso de herbicidas alternativos de ação total, durante o manejo invernal em sistemas de plantio direto, pode constituir uma forma recomendável de manejo em áreas onde as populações de capim-marmelada apresentam resistência aos herbicidas inibidores da ACCase.

Tabela 3 - Porcentagem de controle do capim-marmelada aos 21 DAT, do experimento com herbicidas de manejo

\begin{tabular}{|c|c|c|c|c|}
\hline \multirow{2}{*}{$\begin{array}{l}\text { Tratamentos } \\
\text { (Herbicidas) }\end{array}$} & \multirow{2}{*}{$\begin{array}{c}\text { Doses em g } \\
\left(\text { i.a. ha }{ }^{-1}\right)\end{array}$} & \multicolumn{2}{|c|}{ Biótipos } & \multirow{2}{*}{ Média dos tratamentos } \\
\hline & & $S$ & $\mathrm{R}$ & \\
\hline 1. glyphosate & 1.440 & $100 \mathrm{~A}$ & $100 \mathrm{~A}$ & 100 \\
\hline 2. paraquat & 600 & $100 \mathrm{~A}$ & $100 \mathrm{~A}$ & 100 \\
\hline 3. amônio-glufosinate & 600 & $99,1 \mathrm{~A}$ & $98,7 \mathrm{~A}$ & 98,9 \\
\hline 4. sulfosate & 1.440 & $100 \mathrm{~A}$ & $100 \mathrm{~A}$ & 100 \\
\hline 5. paraquat + diuron & $300+600$ & $100 \mathrm{~A}$ & $100 \mathrm{~A}$ & 100 \\
\hline 6. MSMA + diuron & $980+2.520$ & $76,7 \mathrm{~A}$ & $76,7 \mathrm{~A}$ & 76,7 \\
\hline 7. sethoxydim & 230 & $94,1 \mathrm{~A}$ & $1,9 \mathrm{~B}$ & 44,6 \\
\hline 8. fluazifop-butil & 250 & $89,4 \mathrm{~A}$ & $0,6 \mathrm{~B}$ & 37,5 \\
\hline 9. Testemunha & & $0 \mathrm{~A}$ & $0 \mathrm{~A}$ & 0 \\
\hline \multicolumn{2}{|c|}{ Média dos biótipos } & $84,3 \mathrm{~A}$ & $64,4 \mathrm{~B}$ & \\
\hline
\end{tabular}

Médias dentro de cada tratamento seguidas de mesma letra na linha (letras maiúsculas) não diferem entre si. 


\section{Adjuvantes}

Na avaliação realizada aos 7 DAA no biótipo resistente (Tabela 4), verificou-se que os herbicidas sethoxydim e fenoxaprop não sofreram influência da presença do aditivo, sendo o controle apresentado baixo, próximo a zero. Já o fluazifop proporcionou índices de controle superiores, porém ainda insatisfatórios, no controle da planta daninha, e o aditivo sulfato de amônio melhorou o desempenho deste herbicida em relação ao tratamento sem aditivo.

A porcentagem de controle do biótipo suscetivel para todos os herbicidas foi consideravelmente maior em relação ao resistente (Tabela 4); entretanto, contrariamente ao que aconteceu com o biótipo resistente, o fluazifop proporcionou controle inferior aos herbicidas sethoxydim e fenoxaprop-ethyl na maioria das situações testadas. Dentre os aditivos, verificou-se que a uréia foi melhor que o sulfato de amônio, porém não diferiu do tratamento sem aditivo, para todos os herbicidas, com exceção do fenoxaprop-ethyl.

As diferenças observadas aos 7 DAT (Tabela 4) desapareceram aos 14 DAT (Tabela 5). Dessa forma, verifica-se que o fluazifop apresentou efeito de controle mais lento que o dos demais produtos, mas o controle final foi o mesmo. Conclui-se, em relação aos aditivos, que sua adição à calda não melhorou a atividade dos herbicidas, em nenhum dos biótipos. Assim, o uso de sulfato de amônio ou uréia como forma de incremento da atividade desses herbicidas não é uma alternativa para manejo de resistência. Essas conclusões são confirmadas quando as Tabelas 4 e 5 são analisadas em conjunto. Nestas tabelas fica comprovada a resistência do biótipo de capim-marmelada estudado e confirmada que esta resistência é cruzada aos herbicidas inibidores da ACCase, pertencentes aos grupos químicos dos ariloxifenoxipropionicos (fluazifop e fenoxaprop) e cicloexanodionas (sethoxydim).

Tabela 4 - Avaliação da porcentagem de controle aos 7 DAT para o experimento com adjuvantes

\begin{tabular}{|c|c|c|c|c|c|c|}
\hline \multirow{2}{*}{ Herbicidas } & \multicolumn{3}{|c|}{$\mathrm{R}$} & \multicolumn{3}{|c|}{$\mathrm{S}$} \\
\hline & Uréia & S.A. & S/ Adit. & Uréia & S.A. & S/ Adit. \\
\hline sethoxydim & $0,50 \mathrm{~b} \mathrm{~A}$ & $0,13 \mathrm{~b} \mathrm{~A}$ & $0,84 \mathrm{~b} \mathrm{~A}$ & 92,13 a $\mathrm{A}$ & 71,52 a $\mathrm{B}$ & 81,94 a $\mathrm{AB}$ \\
\hline fluazifop-butil & 22,10 a $\mathrm{AB}$ & 36,20 a $\mathrm{A}$ & 12,88 a B & $66,59 \mathrm{~b} \mathrm{~A}$ & 43,62 b B & $63,00 \mathrm{~b} \mathrm{AB}$ \\
\hline fenoxaprop-ethyl & 8,46 a $\mathrm{A}$ & $1,63 \mathrm{~b} \mathrm{~A}$ & $4,76 \mathrm{ab} \mathrm{A}$ & $77,81 \mathrm{ab} A B$ & $63,03 \mathrm{ab} \mathrm{B}$ & 85,23 a $\mathrm{A}$ \\
\hline Média & \multicolumn{3}{|c|}{$6,63 \mathrm{~B}$} & \multicolumn{3}{|c|}{$72,72 \mathrm{~A}$} \\
\hline
\end{tabular}

- Médias dentro de cada tratamento seguidas de mesma letra na linha (letras maiúsculas) não diferem entre si, assim como as médias seguidas de mesma letra (letras minúsculas) dentro de cada biótipo na coluna.

Tabela 5 - Avaliação da porcentagem de controle aos 14 DAT para o experimento com adjuvantes

\begin{tabular}{|l|c|c|c|c|c|c|}
\hline \multirow{2}{*}{ Herbicidas } & \multicolumn{3}{|c|}{ R } & \multicolumn{2}{c|}{ S } \\
\cline { 2 - 7 } & Uréia & S.A. & S/ Adit. & Uréia & S.A. & S/ Adit. \\
\hline sethoxydim & 2,16 a A & 1,3 b A & 2,96 a A & 99,87 a A & 91,97 a B & 97,72 ab A \\
fluazifop-butil & 5,70 a A & 5,48 ab A & 13,41 a A & 93,10 a A & 87,08 a A & 87,10 b A \\
fenoxaprop-ethyl & 4,03 a A & 13,87 a A & 3,00 a A & 95,70 a A & 78,10 a B & 99,81 a A \\
\hline \multicolumn{1}{|c|}{ Média } & \multicolumn{3}{|c|}{$5,1 \mathrm{~B}$} \\
\hline
\end{tabular}

- Médias dentro de cada tratamento seguidas de mesma letra na linha (letras maiúsculas) não diferem entre si, assim como as médias seguidas de mesma letra (letras minúsculas) dentro de cada biótipo na coluna. 
De acordo com os resultados obtidos nos experimentos conduzidos neste trabalho de pesquisa, conclui-se que a população de capimmarmelada estudada, proveniente de áreas com aplicação repetitiva de herbicidas inibidores da ACCase, com suspeita de seleção de uma população resistente, não apresenta resistência múltipla aos principais herbicidas de manejo usados no plantio direto destas áreas. Estes herbicidas são chamados de manejo, uma vez que não apresentam seletividade à cultura da soja e servem para a formação da palhada para o sistema de plantio direto, constituindo uma forma alternativa de manejo de populações resistentes de capim-marmelada.

No que se refere ao uso de aditivos à calda dos herbicidas inibidores da ACCase, buscando-se maior eficiência de controle para as plantas resistentes a este grupo, de acordo com os resultados, não houve diferença entre os tratamentos herbicidas sem aditivo ou com sulfato de amônio ou com uréia. Portanto, não seria recomendada a mistura desses aditivos nas aplicações em áreas onde o biótipo resistente existe, pois só se estaria adicionando custo às aplicações, sem produzir controle satisfatório.

\section{LITERATURA CITADA}

CRHISTOFFOLETI, P.J.; CORTEZ, M.G.; VICTORIA FILHO, R. Resistance of alexanderweed (Brachiaria plantaginea) to ACCase inhibitor herbicides in soybean from Paraná State, Brazil. In: MEETING OF THE WEED SCIENCE SOCIETY OF AMERICA, Chicago, 1998. Abstract... Chicago: WSSA, 1998. p.65.

CHRISTOFFOLETI, P.J.; VITÓRIA FILHO, R.; SILVA, C.D. Resistência de plantas daninhas aos herbicidas. Planta Daninha, v.12, n.1, p.13-20, 1994.

COSTA, J. From research to practice: staying ahead of the problem. In: DE PRADO, R.; JORRIN, J.; GARCIIA-TORRES, L. Weed crop resistance to herbicides. Córdoba: Kluwer Academic Publishers, 1997. p.316-320.
GAZZIERO, D.L.P.; CHRISTOFFOLETI, P.J.; MACIEL, C.D.M.; SCARAMUZZA Jr., J.R. Resistência de biótipos de Brachiaria plantaginea aos herbicidas inibidores da ACCase aplicados em soja. In: CONGRESSO BRASILEIRO DA CIÊNCIA DAS PLANTAS DANINHAS, 21, 1997, Caxambu. Anais... Caxambu: SBCPD, 1997. p.88.

GILL, G.S. Prevention and control of herbicide resistant weeds in Australia. In: DE PRADO, R., JORRÍN, J., GARCÍA-TORRES, L. Weed crop resistance to herbicides. Córdoba: Kluwer Academic Publishers, 1997. p.305-313.

MATTHEWS, J.M. Management of herbicide resistant weed populations. In: POWLES, HOLTUM, S.B. Herbicide resistance in plants: biology and biochemistry. Adelaide: Lewis Publishers, 1994. p.317-335.

MOSS, S. Strategies for the prevention and control of herbicide resistance in annual grass weeds. In: DE PRADO, R.; JORRÍN, J.; GARCÍATORRES, L. Weed crop resistance to herbicides. Córdoba: Kluwer Academic Publishers, 1997. p.283-290.

OWEN, M.D.K. Risks and benefits of weed management technologies. In: DE PRADO, R.; JORRÍN, J.; GARCÍA-TORRES, L. Weed crop resistance to herbicides crop resistance to herbicides. Córdoba: Kluwer Academic Publishers, 1997. p.291-297.

PONCHIO, J.A.R. Resistência de Bidens pilosa aos herbicidas inibidores da enzima acetolactato sintase. Piracicaba: Escola Superior de Agricultura “Luiz de Queiroz", 1997. 120p. Tese (Doutorado em Agronomia) - Escola Superior de Agricultura "Luiz de Queiroz", 1997.

POWLES, S.B.; HOLTUM, J.A.M. Herbicide resistance in plants: biology and biochemistry. Adelaide: Lewis Publishers, 1994. 353p.

VIDAL, R.A.; FLECK, N.G. Three weed species with confirmed resistance to herbicides in Brazil. In: MEETING OF SCIENCE SOCIETY OF AMERICA, 37, 1997. Abstracts... Chicago: WSSA, 1997. p. 100. 